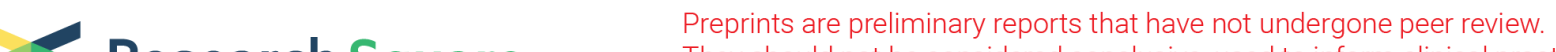 $\begin{array}{ll}\text { Research Square } & \text { They should not be considered conclusive, used to inform clinical practice, } \\ \text { or referenced by the media as validated information. }\end{array}$
}

\section{All-In-One Approaches for Triple-Negative Breast Cancer Therapy: Metal-Phenolic Nanoplatform for MR Imaging-Guided Combinational Therapy}

\section{Qi Xie}

Huazhong University of Science and Technology Tongji Medical College

\section{Shichao Li}

Huazhong University of Science and Technology Tongji Medical College

\section{Xingxing Feng}

Huazhong University of Science and Technology Tongji Medical College

\section{Jingyu Shi}

Huazhong University of Science and Technology Tongji Medical College

\section{Yang Li}

Huazhong University of Science and Technology Tongji Medical College

\section{Guanjie Yuan}

Huazhong University of Science and Technology Tongji Medical College

\section{Conglian Yang}

Huazhong University of Science and Technology

\section{Yaqi Shen}

Huazhong University of Science and Technology Tongji Medical College

\section{Li Kong}

Huazhong University of Science and Technology

Zhiping Zhang ( $\nabla$ zhipingzhang@hust.edu.cn )

Huazhong University of Science and Technology https://orcid.org/0000-0001-9975-4862

\section{Research}

Keywords: Self-assembly, MPNs, Bleomycin, Chemodynamic therapy, MRI

Posted Date: December 29th, 2021

DOI: https://doi.org/10.21203/rs.3.rs-1173894/v1

License: (1) (1) This work is licensed under a Creative Commons Attribution 4.0 International License. Read Full License 


\section{Abstract \\ Background}

Conventional chemotherapy has poor efficacy in triple-negative breast cancer (TNBC) which is highly heterogeneous and aggressive. Imaging-guided therapy is usually combined with diverse treatment modalities, could realize the integration of diagnosis and treatments. Therefore, the primary challenge for combinational therapy is designing proper delivery systems to accomplish multiple synergistic effects.

\section{Results}

Herein, a facile nanoplatform is manufactured to fulfill the all-in-one approaches for TNBC combinational therapy. $\mathrm{Fe}^{3+}$-based metal-phenolic networks (MPNs) with bovine serum albumin (BSA) modification serve as drug delivery carriers to encapsulate bleomycin (BLM), forming BFE NPs@BSA. It is found that BFE NPs@BSA could be further used as photothermal transduction agents and $\mathrm{T}_{1}$-weighted magnetic resonance imaging (MRI) contrast agents. Once internalized into tumor cells, released BLM could cause DNA damage, while Fenton reactions are initiated to produce highly toxic $\cdot \mathrm{OH}$. Upon laser irradiation, $\mathrm{BFE}$ NPs@BSA could convert light into heat to achieve synergistic therapeutic effects. Moreover, as $\mathrm{T}_{1^{-}}$ weighted MRI contrast agents, BFE NPs@BSA could provide diagnosis and treatment monitoring for individualized precise therapy.

\section{Conclusions}

This strategy provides an all-in-one theranostic nanoplatform for MRI-guided combinational therapy against TNBC.

\section{Introduction}

The most common cancer among women is breast cancer, which is the second leading cause of cancer death in women after lung cancer. [1, 2] Triple-negative breast cancer (TNBC), which accounts for $15 \%$ of breast cancer, is highly heterogeneous and aggressive. $[3,4]$ TNBC is prone to recurrence and distant metastasis, resulting in an overall poor prognosis in patients. [5] Therefore, accurate tumor detection is essential to predict the patient's response to treatment and guide treatment strategies, thereby improving the therapeutic effect of TNBC. For example, magnetic resonance imaging (MRI), as a diagnostic tool with noninvasiveness, high resolution and unlimited penetration depth [6], can provide real-time information on the pathological features and progression of tumors. Imaging-guided therapy has the potential to realize the integration of diagnosis and treatments. The current consensus among researchers is that monotherapy is far from adequate to achieve satisfactory therapeutic effects. Therefore, strategies of multiple combinational therapy assisted by imaging modalities have become the primary concerns. 
Among the existing treatment strategies for TNBC, chemotherapy is still one of the main therapeutic options, although patients receiving chemotherapy tend to relapse more frequently and exhibit a worse prognosis. [7-9] As an alternative, dynamic therapy which could damage the cellular components by producing various reactive species such as reactive oxygen species (ROS), has become a promising cancer therapeutic strategy. Reactive species can not only arise from the stimulation of exogenous energy (e.g., light, ultrasound, and ionizing irradiation), but also from the activation of endogenous chemical energy sources such as hydrogen peroxide $\left(\mathrm{H}_{2} \mathrm{O}_{2}\right)$. [10-13] Chemodynamic therapy (CDT) is a specific cancer dynamic therapy that requires no external energy input. CDT initiates intracellular Fenton or Fenton-like reactions, converting $\mathrm{H}_{2} \mathrm{O}_{2}$ into strongly toxic hydroxyl radicals $(\cdot \mathrm{OH})$ under acidic tumor microenvironment (TME), thereby killing cancer cells. The overexpressed $\mathrm{H}_{2} \mathrm{O}_{2}$ in cancer cells serves as the reaction substrate, while transition metal elements such as iron and copper can be used as catalysts. [14] CDT is highly specific to the TME, and therefore exhibits better therapeutic effect and few side effects, compared with conventional chemotherapy. $[15,16]$ However, the mild acidity, low $\mathrm{H}_{2} \mathrm{O}_{2}$ content and overexpressed reducing substances such as glutathione (GSH) in TME sometimes limit the therapeutic efficacy of CDT. [17]

It has been reported that the efficacy and sensitivity of cancer treatment of CDT would be significantly improved when combined with other therapies[18], such as increasing the temperature at tumor site by photothermal therapy (PTT). [19] Elevated temperature could accelerate Fenton or Fenton-like reactions and promote the production of $\cdot \mathrm{OH}$. The rational combination of CDT and PTT can overcome the limitations and shortcomings of monotherapy to achieve synergistic therapeutic effects. Therefore, it is extremely important to design proper CDT reagents with photothermal transduction effects. Moreover, PTT is a powerful and non-invasive cancer treatment with high specificity and accurate spatio-temporal selectivity. Photothermal transduction agents (PTAs) are applied to capture and convert energy from light into heat for PTT, thereby raising the temperature of the local tissues to kill cancer cells. $[20,21]$ It causes less damage to surrounding healthy tissues and could eliminate a wide variety of tumors. In order to enhance therapeutic effect and reduce non-specific light damage to healthy tissues, PTAs are usually assisted by multiple imaging modalities to achieve precise treatment. [22] Reasonable design of therapeutic agents with imaging modalities could provide detailed information for tumor diagnosis and treatment monitoring, which offers new hope for effective TNBC treatment.

Metal ions are extensively applied in biomedical imaging. Iron is an essential trace metal element involved in many physiological processes of human body. [23-25] Recent studies have reported that iron ions and multidentate phenolic ligands (natural polyphenols) could form stable super-molecular network structures through coordination bonds, which are known as metal-phenolic networks (MPNs). [26-29] Due to their intrinsic mechanical stability, thermal stability and $\mathrm{pH}$ responsiveness, MPNs have the potential to be excellent drug delivery vehicles with additional photothermal transduction ability and therapeutic functions. [30-32] For example, $\mathrm{Fe}^{3+}$ ions can serve as contrast agents for MRI on account of the significant $\mathrm{T}_{1}$ contrast enhancement effect. The magnetism of $\mathrm{Fe}^{3+}$ ions depends largely on their coordination state with the ligands. [33-35] MPNs possessed pH-responsive dissociation features, which 
enable MPNs to serve as $\mathrm{T}_{1}$-weighted contrast agents specific for weak acidic TME. Moreover, excessive iron can up-regulate intracellular ROS, inducing tumor cell apoptosis. [23, 36] Exogenous iron element may offer new approaches for effective tumor therapy. Thus $\mathrm{Fe}^{3+}$ ions based-MPNs are capable of serving as theranostic nanoplatforms which simultaneously integrates diagnostics and multiple therapies to realize personalized precise medicine. [32, 37]

Bleomycin (BLM) is a class of glycopeptide antibiotics with potent antitumor activity. It is widely used in the clinical treatment of lymphomas, squamous cell carcinomas and germ cell tumors. [38] On account of the metal-binding domain, BLM could bind to transition metals ( $\mathrm{Fe}^{\mathrm{II}}$ or $\mathrm{Cu}^{\prime}$ ) and form BLM-metal complexes. In the presence of single-electron reductants and oxygen $\left(\mathrm{O}_{2}\right)$, BLM-metal complexes were converted into activated forms, producing superoxide and hydroxyl radicals, that resulted in DNA cleavage and damage. $[39,40]$ However, BLM has severe dose-dependent pulmonary toxicity, which limits its clinical application. Pulmonary toxicity occurs in approximately $20 \%$ of patients treated with BLM, with pulmonary fibrosis being the most severe form. [41] Owing to its relatively large molecular weight (1.5 $\mathrm{kDa}$ ) and hydrophilicity, BLM has very poor membrane permeability, resulting in low intracellular concentrations. [42-45] Hence, it is urgent to develop rational drug delivery systems for better therapeutic efficacy and lower side effects.

Herein, we proposed a MPNs-based nanoplatform to accomplish the all-in-one approach for TNBC combinational therapy (Scheme 1). On the whole, MPNs formed by epigallocatechin gallate (EGCG, tea polyphenol) and $\mathrm{Fe}^{3+}$ could effectively encapsulate BLM to prepare BFE NPs by self-assembly. In order to improve the stability of nanoparticles and mask the strong adhesion of polyphenols, which may lead to strong protein interactions in bloodstream, $[46,47]$ bovine serum albumin (BSA) was coated on the surface of BFE NPs, forming BFE NPs@BSA. In our design, as $\mathrm{T}_{1}$-weighted MRI contrast agents, $\mathrm{Fe}^{3+}$ ions could provide diagnosis and treatment monitoring for individualized precise therapy. We further investigated both in vitro and in vivo therapeutic efficacy of BFE NPs@BSA on murine TNBC cell line (4T1) and tumor xenograft model. Once internalized into tumor cells, $\mathrm{Fe}^{3+}$ ions were reduced into $\mathrm{Fe}^{2+}$ ions by GSH. Then $\mathrm{Fe}^{2+}$ and BLM initiated Fenton reactions to produce highly toxic $\cdot \mathrm{OH}$, leading to $\mathrm{GSH}$ depletion and ROS amplification. As a chemotherapeutic agent, activated BLM could cause DNA damage. Upon laser irradiation, BFE NPs@BSA converted light into heat to ablate tumor. Furthermore, elevated local temperature contributed to accelerated Fenton reactions. This strategy provided an all-in-one theranostic nanoplatform for MRI-guided synergistic photothermal and chemodynamic therapy against TNBC.

\section{Experimental Section}

\section{Materials}

BLM was purchased from Acmec biochemical (China). Bovine serum albumin (BSA) and EGCG were purchased from Macklin (China). $\mathrm{FeCl}_{3} \cdot 6 \mathrm{H}_{2} \mathrm{O}$ was purchased from Aladdin (China). Phosphate buffer 
saline (PBS) was purchased from Servicebio (China). Penicillin-streptomycin solution (100x), fetal bovine serum (FBS), RPMI medium 1640, and trypsin were purchased from Gibco (USA). 4',6-diamidino-2phenylindole (DAPI), 1,1'-dioctadecyl-3,3,3'-diotetramethylindotricarbocyanine iodide (DiR) and ROS assay kit were obtained from Beyotime (China). Ethanol absolute, ammonia solution, ethylenediamine tetraacetic acid (EDTA), urea, glucose and all other chemical reagents were purchased from Sinopharm Chemical Reagent Co., Ltd (China).

\section{Cell culture and animal model}

Murine TNBC cell line (4T1) was cultured with RPMI medium 1640 (containing 10\% FBS, $100 \mathrm{lU} / \mathrm{mL}$ of penicillin and $100 \mu \mathrm{g} / \mathrm{mL}$ of streptomycin) in a humidified atmosphere incubator with $5 \% \mathrm{CO}_{2}$ at $37{ }^{\circ} \mathrm{C}$. Female BALB/c mice (6 to 8 weeks) were purchased from the Laboratory Animal Resources of Huazhong University of Science and Technology (HUST) and raised under specific pathogen-free (SPF) conditions in the Animal Center of HUST. All animal experiments were reviewed and approved by the Experimental Animal Committee of HUST. 4T1 tumor xenograft model was established as follows: the suspension of $4 \mathrm{~T} 1$ cells $\left(2 \times 10^{5}\right.$ cells in $200 \mu \mathrm{L}$ PBS $)$ were inoculated under the flank skin of mice. The volume of tumors was calculated as $V=L \times W^{2} / 2$ ( $L$ : length of tumor; $W$ : width of tumor).

\section{Preparation and optimization of nanoparticles}

Preparation of BFE NPs Firstly, BFE NPs were prepared by BLM, $\mathrm{FeCl}_{3} \cdot 6 \mathrm{H}_{2} \mathrm{O}$ and EGCG. BLM $(0.3 \mathrm{mg})$ was dissolved in water $(50 \mu \mathrm{L})$ and mixed with $\mathrm{FeCl}_{3} \cdot 6 \mathrm{H}_{2} \mathrm{O}(0.3 \mathrm{mg})$ which was dissolved in water $(15 \mu \mathrm{L})$ at room temperature. After stirring for $5 \mathrm{~min}$, the mixture of $\mathrm{BLM}$ and $\mathrm{FeCl}_{3} \cdot 6 \mathrm{H}_{2} \mathrm{O}$ was dispersed in ethanol absolute $(1 \mathrm{~mL})$ which contained $50 \mu \mathrm{L}$ of EGCG solution $(6 \mathrm{mg} / \mathrm{mL})$. After stirring for $5 \mathrm{~min}, \mathrm{NH}_{3} \cdot \mathrm{H}_{2} \mathrm{O}(30$ $\mu \mathrm{L})$ was added to the mixture solution. Subsequently, the products were centrifuged (14000 rpm) for 10 min at $4{ }^{\circ} \mathrm{C}$ to collect precipitation. The obtained precipitation (BFE NPs) was redispersed in $1 \mathrm{~mL}$ of water and sonicated for $1 \mathrm{~min}$. To explore the optimal ratio of EGCG, the amount of $\mathrm{BLM}$ and $\mathrm{Fe}^{3+}$ was fixed, the preparation process was the same as the above method at different molar ratios (BLM:EGCG:Fe ${ }^{3+}=1: X: 6, X=0,1,2,3$, and 4). Similarly, the amount of BLM and EGCG was fixed, the nanoparticles were prepared at different molar ratios (BLM:EGCG:Fe ${ }^{3+}=1: 3: Y, Y=0,2,4,6$, and 8) to explore the optimal ratio of $\mathrm{Fe}^{3+}$.

In vitro stability study In order to test the stability of nanoparticles in different media, BFE NPs were dispersed in ultrapure water $\left(\mathrm{H}_{2} \mathrm{O}\right), 5 \%$ glucose solution (Glu), normal saline (NS), phosphate buffer (PB), or PBS, respectively. After $24 \mathrm{~h}$, the suspensions were photographed and measured. Furthermore, BFE NPs were dispersed in $100 \%$ FBS, DMEM (cell culture medium) or DMEM containing 10 or $50 \%$ FBS (denoted as 10 or $50 \%$ FBS-M), respectively. The long-term stability of nanoparticles was performed by storing the nanoparticles in ultrapure water, PBS and complete medium (RPMI medium 1640 containing $10 \%$ FBS, penicillin and streptomycin, denoted as $10 \%$ FBS-M) at $37{ }^{\circ} \mathrm{C}$ for 7 days. The particle size of nanoparticles was recorded at the corresponding time points. 
Preparation of BFE NPs@BSA In the following step, BFE NPs were further incubated in BSA solution (0, 1, $2,5,10$ and $15 \mathrm{mg} / \mathrm{mL}$ ) with gentle stirring for $1 \mathrm{~h}$. The product was centrifuged at $14000 \mathrm{rpm}$ for $30 \mathrm{~min}$ $\left(4{ }^{\circ} \mathrm{C}\right)$ to remove free albumin and washed twice by repeated centrifugation. The precipitation was redispersed in $1 \mathrm{~mL}$ of water/PBS and sonicated for $1 \mathrm{~min}$. The albumin-coated BFE NPs were denoted as BFE NPs@BSA.

\section{Characterization and spectra of nanoparticles}

The hydrodynamic diameter, polydispersity index (PDI) and zeta potential of nanoparticles were measured by dynamic light scattering (DLS) by Zeta Potential Analyzer (Zeta PALS, Brookhaven Instruments, USA). Furthermore, the drug encapsulation efficiency (EE) of BLM and $\mathrm{Fe}^{3+}$ were detected by high performance liquid chromatography (HPLC) and atomic absorption spectrometry (AAS), respectively. Ultraviolet spectrophotometer (Lambda 365, PerkinElmer, USA), fluorescence spectroscopy analyzer (F4600, Hitachi, Japan) and Fourier transform infrared spectroscopy (FT-IR) analyzer (AVATAR 360, Thermo, USA) were used for analyzing the properties and assembly mechanisms of BFE NPs@BSA. Transmission Electron Microscope (TEM, JEM-1230, Japan) was used to characterize the morphology of BFE NPs and BFE NPs@BSA.

\section{Self-assembly mechanisms study}

BFE NPs and BFE NPs@BSA were dispersed in PBS, 0.9\% NaCl solution, 5 M urea solution, 100 mM EDTA solution and $10 \%$ sodium dodecyl sulfate (SDS) solution, respectively. After $24 \mathrm{~h}$, the suspensions were photographed and recorded.BFE NPs@BSA solution was treated with EDTA, and the fluorescence intensity of BFE NPs@BSA was recorded with excitation wavelength at 308 nm after different incubation.

\section{In vitro $\mathrm{pH}$ sensitive study}

To further investigate the pH sensitivity of BFE NPs@BSA, 0.5 mL of BFE NPs@BSA was dispersed in 4 $\mathrm{mL}$ PBS with different $\mathrm{pH}$ values $(7.4,6.5$ or 5.5$)$. The fluorescence intensity of BFE NPs@BSA was recorded with excitation wavelength at $308 \mathrm{~nm}$ and the particle size of BFE NPs@BSA with different incubation time was also measured.

\section{In vitro drug release}

To monitor the release of BLM from BFE NPs@BSA, the nanoparticles were suspended in $1 \mathrm{~mL}$ of PBS with $\mathrm{pH}$ values of $7.4,6.5$ and 5.5 , respectively. All samples were further placed in a constant temperature vibration incubator at $37^{\circ} \mathrm{C}(100 \mathrm{rpm})$. The supernatant was collected as the released medium at different time points by centrifugation at 10000 rpm for 15 min. The BFE NPs@BSA precipitation was redispersed in equal volume fresh medium and kept at the same conditions. The content of BLM in release medium was determined by HPLC. The operation of experiment was repeated three times.

\section{In vitro photothermal evaluation}


To assess the photothermal performance of BFE NPs and BFE NPs@BSA, the solution with different Fe $\mathrm{F}^{3+}$ concentrations $(0.2,0.4$ and $0.8 \mathrm{mM})$ was placed in a centrifuge tube $(1.5 \mathrm{~mL})$ to receive $808 \mathrm{~nm}$ laser irradiation with different power density of $2.0,2.5,3.0 \mathrm{~W} / \mathrm{cm}^{2}$ for $10 \mathrm{~min}$. The temperature variations were recorded using a digital infrared thermometer at designed time points.

\section{In vitro cytotoxicity assay}

MTT assay The cytotoxicity of BFE NPs@BSA was performed on 4T1 cell line. The cells were seeded in 96-well plates at a density of $1 \times 10^{4}$ cells/well and cultured at $37^{\circ} \mathrm{C}$ for $12 \mathrm{~h}$. Free BLM and BFE NPs@BSA at different BLM concentrations $(0,1,2,4$, and $8 \mu \mathrm{M})$ were added into cells and further incubated for another $6 \mathrm{~h}$. Then, the cells were washed with PBS three times, the group of BFE NPs@BSA+L were irradiated under the $808 \mathrm{~nm}$ laser $\left(2.5 \mathrm{~W} / \mathrm{cm}^{2}\right)$ for $2 \mathrm{~min}$. After another $18 \mathrm{~h}, 10 \mu \mathrm{L}$ of MTT $(5 \mathrm{mg} / \mathrm{mL})$ solution was added to each cell well and incubated for $4 \mathrm{~h}$. Cell medium was removed before adding DMSO $(150 \mu \mathrm{L})$ to dissolve the formazan crystals. To determine cell viability, absorbance at $490 \mathrm{~nm}$ was measured. The cell viability was calculated as follows:

Cell viability $(\%)=\left(O D_{\text {sample }}-O D_{\text {blank }} / O D_{\text {control }}-O D_{\text {blank }}\right) \times 100 \%$

Live/dead staining assay The cells were seeded in 24-well plates at a density of $1 \times 10^{5}$ cells/well and cultured at $37^{\circ} \mathrm{C}$ for $12 \mathrm{~h}$. Free BLM and BFE NPs@BSA at BLM concentration of $8 \mu \mathrm{M}$ were added into cells and further incubated for another $6 \mathrm{~h}$. Then, the cells were washed with PBS three times, the group of Laser and BFE NPs@BSA+L were irradiated under the $808 \mathrm{~nm}$ laser $\left(2.5 \mathrm{~W} / \mathrm{cm}^{2}\right)$ for $2 \mathrm{~min}$. After another $18 \mathrm{~h}$, the live and dead cells were stained with calcein AM and PI according to the manufacturer's instructions. The cells were then washed with cold PBS and observed by fluorescence microscope (CKX53, Olympus, Japan).

\section{In vitro cellular uptake study}

Coumarin-6 loaded BFE NPs (BFE NPs ${ }_{\mathrm{C} 6}$ ) and BFE NPs@BSA (BFE NPs@BSA ${ }_{C 6}$ ) were prepared for cellular uptake study, respectively. 4T1 cells were co-cultured with BFE NPs $\mathrm{C}_{6}$ or BFE NPs@BSA $\mathrm{C}_{\mathrm{C} 6}$ (at coumarin- 6 content of $0.5 \mu \mathrm{g} / \mathrm{mL}$ ) for $2 \mathrm{~h}$ or $6 \mathrm{~h}$. The nucleus was stained by DAPI after incubation. The cellular uptake images were acquired by fluorescence microscopy (CKX53, Olympus, Japan).

\section{In vitro ROS generation}

4T1 cells were seeded in 6-well plates and cultured overnight. The medium was replaced with fresh medium containing free BLM $(4 \mu \mathrm{M})$, BFE NPs@BSA $(4 \mu \mathrm{M})$, or blank medium. After incubation for $8 \mathrm{~h}$, the cells were washed with PBS three times. Then, the cells were irradiated under the $808 \mathrm{~nm}$ laser $(2.5$ $\mathrm{W} / \mathrm{cm}^{2}$ ) for $15 \mathrm{~s}$ (this group was denoted as BFE NPs@BSA+L). The cells after different treatments were incubated with DCFH-DA at $37^{\circ} \mathrm{C}$ for $30 \mathrm{~min}$. Then, the cells were washed with PBS to remove free probe, following by observation via fluorescence microscopy and analysis via flow cytometry. 


\section{In vitro relaxivity measurements and MRI performance of BFE NPs@BSA}

The relaxivity measurements of nanoparticles were carried out on a 3.0 T clinical MRI instrument (Skyra, Siemens Healthcare, Erlangen, Germany). BFE NPs@BSA with different concentration gradients of Fe (0, $0.1,0.2,0.3,0.4$, and $0.5 \mathrm{mM}$ ) were prepared and measured. The parameters for $T_{1}$ measurements were set as follows: inversion time $(\mathrm{TI})=30,60,90,120,150,250,400,600,800,1200,1600,2000,2400,2800$, and $3200 \mathrm{~ms}$; repetition time $(T R)=1500 \mathrm{~ms}+\mathrm{Tl}$; echo time $(\mathrm{TE})=15 \mathrm{~ms}$; number of excitations (NEX) = 8. The parameters for $T_{2}$ measurements were set as follows: $T R=3000 \mathrm{~ms}$; TE was between $20 \mathrm{~ms}$ and $600 \mathrm{~ms}$ with an interval of $20 \mathrm{~ms}$; NEX $=8$. The following parameters were maintained for all measurements: slice thickness $=5 \mathrm{~mm}$; field-of-view $(\mathrm{FOV})=120 \times 120 \mathrm{~mm}$. For in vitro imaging, the series of BFE NPs@BSA solutions with different Fe concentrations were prepared and sealed in $2.0 \mathrm{~mL}$ Eppendorf tubes for further test. Ultrapure water was set as the blank control. The parameters for $\mathrm{T}_{1}$ weighted images were set as follows: TR $=398 \mathrm{~ms}, \mathrm{TE}=14 \mathrm{~ms}, \mathrm{FOV}=120 \times 120 \mathrm{~mm}^{2}$, matrix size $=256$ $\times 256$, slice thickness $=1 \mathrm{~mm}$, and NEX $=8$. Ultrapure water was set as the blank control.

\section{In vivo MRI study}

The in vivo MR images of tumors were acquired on a 3.0 T clinical MRI instrument (Skyra, Siemens Healthcare, Germany) with an 8-channel 5-cm Rx custom-design coil. Female BALB/c mice bearing 4T1 tumor xenografts were anesthetized and then BFE NPs@BSA (1.1 mg Fe ${ }^{3+}$ per kilogram body weight) were injected through tail vein. $T_{1}$-weighted images were acquired at designed time points. The detailed parameters for in vivo $T_{1}$ imaging were set as follows: $T R=398 \mathrm{~ms}, \mathrm{TE}=17 \mathrm{~ms}, \mathrm{FOV}=40 \times 40 \mathrm{~mm}^{2}$, matrix size $=256 \times 256$, slice thickness $=1 \mathrm{~mm}$, and NEX $=8$. The mice were anesthetized with $1 \%$ isoflurane delivered via a nose cone during the imaging sessions.

\section{Biodistribution study of BFE NPs@BSA}

DiR loaded BFE NPs@BSA (BFE NPs@BSA $A_{\text {DiR }}$ ) was prepared to evaluate biodistribution behavior. The 4T1 tumor-bearing mice were randomly divided into two groups (3 mice in each group). The mice were intravenously injected with $200 \mu \mathrm{L}$ of free DiR or BFE NPs@BSA $A_{\text {DiR }}(0.5$ mg DiR per kilogram body weight). After intravenous injection, the tumors and main organs were obtained and imaged (IVIS Lumina XR system, Trilogy, LI-COR) at designed time intervals.

\section{In vivo dose exploration of BLM}

When the tumor volume grew to about $100 \mathrm{~mm}^{3}$, the $4 \mathrm{~T} 1$ tumor-bearing mice were randomly divided into four groups $(n=5)$ and treated with BFE NPs@BSA at different BLM dosages $(2.5,5$, and $10 \mathrm{mg}$ per kilogram body weight). On the 5th, 8th and 11th day, mice were intravenously injected with $200 \mu \mathrm{L}$ PBS or BFE NPs@BSA at different dosages. The body weight and tumor volume of each mouse were measured and recorded every other day. On the 21th day, all mice were sacrificed to harvest the tumors and major 
organs (heart, liver, spleen, kidneys, and lung), which were weighted and then preserved in $4 \%$ paraformaldehyde solution for histological analysis.

\section{In vivo anti-tumor combination therapy}

The 4T1 tumor-bearing mice were randomly divided into four groups $(n=8)$ when the tumor volume grew to about $100 \mathrm{~mm}^{3}$. On the 6th, 9th and 12th day, mice were intravenously injected with $200 \mu \mathrm{L}$ PBS or BLM, BFE NPs@BSA, BFE NPs@BSA + Laser (BFE NPs@BSA+L) at the same dosage ( $5 \mathrm{mg} / \mathrm{kg})$. For the BFE NPs@BSA $+\mathrm{L}$ group, the tumor sites of mice were irradiated for 2 min at $12 \mathrm{~h}$ post-injection (808 $\mathrm{nm}$ laser at $2.5 \mathrm{~W} / \mathrm{cm}^{2}$ ). The body weight and tumor volume of each mouse were measured and recorded every other day. On the 21st day, all mice were sacrificed to harvest the tumors and major organs (heart, liver, spleen, kidneys, and lung), which were weighted and then preserved in $4 \%$ paraformaldehyde solution for histological analysis.

\section{Statistical analysis}

The data were presented in the form of mean \pm standard deviation (SD). Statistical significance of experimental and control groups was analyzed by one-way ANOVA test at a significance level of $p<0.5$ $\left.(*), p<0.01{ }^{(*}\right)$ and $p<0.001(\star \star \star)$.

\section{Results And Discussions}

\section{Preparation and optimization of nanoparticles}

Even though EGCG and $\mathrm{Fe}^{3+}$ ions have been widely used to prepare MPNs, there were rare reports about using MPNs as carriers for delivery of BLM. Owing to the presence of metal-binding domain in BLM, $\mathrm{Fe}^{3+}$ ions could assemble with BLM in water, forming the core of nanoparticles.[38, 39] Then redundant $\mathrm{Fe}^{3+}$ ions further coordinated with EGCG at the interface of ethanol/water, composing MPNs (Fig. 1A and Additional file 1: Fig. S1). We firstly investigated the proportion of three components required for the formation of BFE NPs. At beginning, the proportion of EGCG was explored by varying its input with fixed amount of $\mathrm{BLM}$ and $\mathrm{Fe}^{3+}$. As shown in Fig. 1B and Additional file 1: Fig. S2A, when the molar ratio between BLM, EGCG and $\mathrm{Fe}^{3+}$ was set at 1:3:6, the corresponding nanoparticles exhibited the darkest color, whereas the particle size reached minimum and the zeta potential also decreased to the lowest. Similarly, the influence of the amount of $\mathrm{Fe}^{3+}$ on BFE NPs was examined. Using particle size and zeta potential as indicators, it has been further confirmed that the prepared nanoparticles were most suitable when the proportion of three components was 1:3:6 (Fig. 1C and Additional file 1: Fig. S2B). Therefore, the molar ratio between BLM, EGCG and $\mathrm{Fe}^{3+}$ in the formation of BFE NPs was fixed at 1:3:6 in the following investigation.

The stability of nanoparticles is a prerequisite for subsequent application and storage. In order to simulate different biological conditions, the particle size changes of BFE NPs incubated in different 
media ( $\mathrm{H}_{2} \mathrm{O}$, Glu, NS, PB, and PBS) were monitored. After $24 \mathrm{~h}$, BFE NPs were quite stable in both water and glucose solution, as the particle size of BFE NPs remained unchanged (Fig. 1D and 1E). But the particle size of BFE NPs increased exponentially in NS, PB and PBS, and sedimentation was even observed in PB and PBS. Furthermore, there was no precipitation of BFE NPs in 10 or 50\% FBS-M or $100 \%$ FBS, and the particle size of nanoparticles slightly increased and then stayed steady (Additional file 1: Fig. S3). Thus, we hypothesized that BFE NPs might adsorb proteins from the serum, which facilitated the stability of nanoparticles. In long-term stability study, BFE NPs were steady and remained the similar size for 7 days in the complete medium containing 10\% FBS (Fig. 1F and Additional file 1: Fig. S4A). In brief, BFE NPs aggregated in various salt solutions, but they exhibited colloidal stability in serum-containing solution, indicating that serum proteins might protect BFE NPs from aggregation.

Polyphenols are highly adherent and exhibit multiple interactions (e.g., hydrogen bonds, hydrophobic, and electrostatic interactions), which allow them to form robust conjugates with other substances. BSA has been widely used to modify nanoparticles to improve the stability. In order to improve the stability of BFE NPs, BFE NPs were further modified with BSA by incubation in BSA solution through surface adsorption (Fig. 1G). When the concentration of BSA reached $5 \mathrm{mg} / \mathrm{mL}$ (Fig. $1 \mathrm{H}$ ), the nanoparticles could remain stable in PBS. Subsequently, with the concentration of BSA continually increased, the particle size of nanoparticles gradually increased. Therefore, the BSA concentration of $5 \mathrm{mg} / \mathrm{mL}$ was selected to prepare BFE NPs@BSA. As expected, the particle size of BFE NPs@BSA remained constant for 7 days in all media, including $\mathrm{H}_{2} \mathrm{O}$, PBS and 10\% FBS-M (Fig. $1 \mathrm{l}$ and Additional file 1: Fig. S4B). The introduction of BSA endowed nanoparticles with great physiological stability and long-term stability, which laid a sufficient foundation for subsequent application.

To sum up, BLM + Fe ${ }^{3+}$ or BLM + EGCG could form nanoparticles (BF NPs or BE NPs). But only when three components were all present, nanoparticles with desired structure could be fabricated. As shown in Fig. 1J, BFE NPs which were prepared in optimal proportion displayed the particle size of $158.9 \pm 1.8 \mathrm{~nm}$ and the zeta potential of $-38.1 \pm 3.5 \mathrm{mV}$ and presented a dark black appearance. The negative surface charge of BFE NPs indicated the structure of MPNs. After BSA coating on the surface of nanoparticles, BFE NPs@BSA remained the black appearance, while their particle size increased by 15 nm $(173.0 \pm 1.4$ $\mathrm{nm}$ ) than BFE NPs and zeta potential was back to $-11.5 \pm 1.1 \mathrm{mV}$. As a result of the masking of BSA, BFE NPs@BSA were less charged, which remarkably improved the stability of nanoparticles.

\section{Characterization and spectra of nanoparticles}

The morphology of nanoparticles was studied by TEM. As shown in Fig. 1K-L and Additional file 1: Fig. S5, BFE NPs possessed network-like structures, which indicated the successful formation of MPNs. While the images of BFE NPs@BSA presented scattered particles because BSA facilitated the dispersion of nanoparticles. As shown in Additional file 1: Table S1, the EE of BLM and Fe ${ }^{3+}$ in BFE NPs@BSA was $65.0 \%$ and $77.2 \%$ ( $80.4 \%$ and $94.9 \%$ in BFE NPs), respectively, indicating this delivery system realized efficient drug packaging. In order to confirm the formation of nanoparticles, the UV-vis spectra, fluorescent spectra and FT-IR spectra were subsequently applied to analyze the formation mechanism. In 
Fig. 2A, the maximum absorption wavelength of BLM at $291 \mathrm{~nm}$ was red-shifted to $297 \mathrm{~nm}$ in the UV-vis spectrum of BFE NPs@BSA, which manifested the $\pi-\pi$ interactions between BLM, EGCG and BSA. Furthermore, BFE NPs@BSA and direct mixture of EGCG+Fe ${ }^{3+}$ exhibited broad absorption in the NIR window, confirming that $\mathrm{Fe}^{3+}$-based MPNs had the ability to serve as photothermal reagents. In Fig. 2B, BLM exhibited a fluorescence emission from 330 to $480 \mathrm{~nm}$ with excitation wavelength at $308 \mathrm{~nm}$ in its fluorescent spectrum. However, the fluorescence of BLM was quenched in BFE NPs@BSA as a result of the formation of coordination bonds between EGCG and Fe ${ }^{3+}$. In Fig. 2C, the -OH stretching bands (3556, 3479 and $3357 \mathrm{~cm}^{-1}$ ) of pure EGCG were not observed in the FT-IR spectrum of BFE NPs@BSA, demonstrating the formation of coordination bonds. BFE NPs@BSA exhibited the characteristic amide I and amide II band vibrations (1648 and $1550 \mathrm{~cm}^{-1}$ ) of BLM and BSA, indicating the existence of BLM and BSA in nanoparticles. All of the above results proved the successful preparation of BFE NPs@BSA.

\section{Self-assembly mechanism study of BFE NPs and BFE NPs@BSA}

In order to further confirm the self-assembly mechanism of BFE NPs and BFE NPs@BSA, the prepared nanoparticles were dispersed into different solutions ( $\mathrm{NaCl}$, urea, EDTA and SDS) to determine the role of electrostatic force, hydrogen bonds, coordination bonds and hydrophobic force, respectively. In Fig. 2D, when BFE NPs were dispersed into $0.9 \% \mathrm{NaCl}$ solution (electrostatic force-eliminating agents), a small amount of precipitation was observed, indicating there were certain electrostatic interactions within nanomedicines. Changes and precipitation were more obvious when BFE was mixed with urea solution which could deconstruct hydrogen bonds, suggesting the hydrogen bonds were involved in the formation of BFE NPs. However, it was found that SDS did not affect nanoparticles, suggesting hydrophobic force barely existed during the assembly process of BFE NPs. The most obvious change of BFE NPs was observed in appearance, where precipitate formed immediately, upon mixing with EDTA solution (coordination bonds-eliminating agents). These results implied that EDTA was the most effective treatment to disintegrate the BFE NPs and coordination force played the main force in the formation of BFE.

To sum up, we could speculate different forces that participated in the formation of nanoparticles as follow: coordination bonds > hydrogen bonds > electrostatic force $\gg$ hydrophobic force. Furthermore, when the BFE NPs@BSA were placed in the above solution, the changes were smaller than that of BFE NPs in appearance. This indicated that the presence of BSA could improve the stability of nanoparticles to a certain degree. It has been proved that the fluorescence of BLM in BFE NPs@BSA was quenched, owing to coordination between $\mathrm{BLM}$ and $\mathrm{Fe}^{3+}$ (Fig. 2B). Therefore, we further investigated the fluorescence recovery ability of BLM from BFE NPs@BSA by adding EDTA solution. The degradation of BFE NPs@BSA was supposed to induce the release of BLM, leading to fluorescence recovery. As shown in Fig. 2E, upon adding EDTA, the fluorescence of BLM gradually recovered and fluorescence intensity gradually increased with incubation time. It indicated that EDTA could destroy the structures of BFE NPs@BSA and lead to the release of BLM. It further confirmed that coordination force played a vital role in the self-assembly process of nanoparticles. 


\section{In vitro $\mathrm{pH}$ sensitive study}

The BFE@BSA was designed with the characteristics of pH-responsive degradation in an acidic environment, owing to the $\mathrm{pH}$ responsiveness of coordination force and hydrogen bonding. In acidic conditions, the disassemble of BFE NPs@BSA was accompanied by BLM release in the acidic TME. To evaluate the pH-responsive capability of BFE NPs@BSA, the size distribution of nanomedicines was measured firstly through dispersing them into buffer solution with $\mathrm{pH}$ at 6.5 and 5.5. From the picture and results in Fig. 2F, it could be seen that the same precipitation occurred in acidic solutions as the addition of EDTA. The particle size of BFE NPs@BSA remained steady with negligible changes at pH 7.4. But at pH 6.5 and 5.5, BFE NPs@BSA became larger than before. Obvious sedimentation and stratification were observed both at $\mathrm{pH} 6.5$ and 5.5, indicating that the nanoparticles were $\mathrm{pH}$-responsive.

Furthermore, the fluorescence recovery ability of BLM from BFE NPs@BSA under acidic conditions (pH 6.5 and 5.5) was also investigated. As expected, the fluorescence intensity of BLM increased gradually with incubation time at both $\mathrm{pH}$ values in Fig. $2 \mathrm{G}$ and $2 \mathrm{H}$. Moreover, the fluorescence intensity of BLM recovered more quickly at $\mathrm{pH} 5.5$ than that at pH 6.5. These results implied that BFE NPs@BSA could gradually dissociate and release the BLM in an acidic environment

\section{In vitro drug release}

To accurately access the BLM release behavior from BFE NPs@BSA in the physiological condition, tumor sites and intracellular acidic environment, in vitro drug release tests were performed in PBS solutions with $\mathrm{pH} 7.4, \mathrm{pH} 6.5$ and pH 5.0, to simulate certain conditions. As shown in Fig. 2l, the amount of BLM released from BFE NPs@BSA was gradually increased as the pH of releasing medium decreased from 7.4 to 5.5. At pH 7.4, the amount of BLM released within the entire experimental period $(72 \mathrm{~h})$ was around $30 \%$, while BLM released was up to $43 \%$ in PBS solution at $\mathrm{pH} 5.5$. The $\mathrm{pH}$ sensitive release profile was mainly ascribed to the dissociation of the coordination bands in BFE NPs@BSA in acidic environment.

\section{In vitro photothermal evaluation}

In our previous study, it was found that $\mathrm{Fe}^{3+}$ ions-based MPNs exhibited a broad absorption in the NIR region, which indicated that the designed reagents might have a photothermal transduction effect. To test the photothermal conversion efficiency of BFE NPs@BSA, the temperature differences $(\Delta T)$ of BFE NPs@BSA solution was checked under different $\mathrm{Fe}^{3+}$ concentrations or power densities. In Fig. 2J-K and Additional file 1: Fig. S6A-B, with the increase of laser intensity (from $2.0 \mathrm{~W} / \mathrm{cm}^{2}$ to $3.0 \mathrm{~W} / \mathrm{cm}^{2}$ ) or iron ions concentration (from $0.2 \mathrm{mM}$ to $0.8 \mathrm{mM}$ ), the temperature of BFE NPs@BSA solution increased significantly. The temperature of BFE NPs@BSA with $\mathrm{Fe}^{3+}$ concentration at $0.8 \mathrm{mM}$ could increase up to $48.1{ }^{\circ} \mathrm{C}$ with temperature change about $21^{\circ} \mathrm{C}$ after irradiation for $10 \mathrm{~min}\left(808 \mathrm{~nm}\right.$ laser at $\left.2.5 \mathrm{~W} / \mathrm{cm}^{2}\right)$. These results suggested that BFE NPs@BSA could serve as effective PTAs.

To further investigate the photothermal stability of nanomedicines, BFE NPs and BFE NPs@BSA (0.8 $\mathrm{mM}$ ) were irradiated for 5 cycles of laser on (808 $\mathrm{nm}$ laser at $2.5 \mathrm{~W} / \mathrm{cm}^{2}, 10 \mathrm{~min}$ ) and laser off (10 min), 
respectively. After 5 cycles, the $\Delta T$ between adjacent peaks was within $0.5^{\circ} \mathrm{C}$ according to Fig. $2 \mathrm{~L}$. It indicated the excellent photothermal stability of both BFE NPs and BFE NPs@BSA under repeated NIR laser irradiation, while coating with BSA did not affect the photothermal conversion efficiency of nanoparticles.

\section{In vitro cytotoxicity assay}

The cell viability was firstly determined by MTT assay to investigate the in vitro anti-tumor ability of BFE NPs@BSA. 4T1 cells were incubated with free BLM or BFE NPs@BSA at different BLM concentrations. As shown in Fig. 3A, both free BLM and BFE NPs@BSA showed weak cytotoxicity at low concentrations. The cell viability of BFE NPs@BSA at BLM concentration of $8 \mu \mathrm{M}$ was $53.2 \%$, which was lower than that of free BLM (66.5\%). And the cell viability of BFE NPs@BSA+L (BLM: $8 \mu \mathrm{M})$ was $47.1 \%$, indicating that BFE NPs@BSA could efficiently kill tumor cells with laser irradiation. Then, live/dead staining assay was conducted by staining cells with Calcein-AM and PI after respective treatments. Calcein-AM (green) could easily penetrate live cell membrane to mark live cells, while PI (red) could only reach the cell nucleus through the disordered membrane of dead cell. As shown in Fig. 3B, the cells which were treated with laser alone showed almost no apparent red fluorescence. More red fluorescent signals were found in the groups treated with free BLM or BFE NPs@BSA (BLM: $8 \mu \mathrm{M})$, and cells treated with BFE NPs@BSA+L presented obvious red fluorescence. All the results illustrated the synergistic cytotoxicity of combination therapy.

\section{In vitro cellular uptake study}

It was reported that the cellular uptake of nanomedicines was significantly influenced by their surface physicochemical characteristics. In order to understand whether the presence of BSA in our system would facilitate the cellular uptake, we evaluated the effect of BSA modification on the in vitro cellular uptake. Before that, green fluorescent dye, coumarin-6, was loaded into BFE NPs during the preparation for fluorescent observations. The internalization of BFE NPs and BFE NPs@BSA into 4T1 cells was observed by fluorescence microscopy. The blue area was the nuclei of $4 \mathrm{~T} 1$ cells stained by DAPI and the green fluorescence resulted from coumarin-6 loaded nanoparticles. As shown in Fig. 3C, the amount of phagocytosed nanoparticles, no matter BFE NPs or BFE NPs@BSA, both increased with time, as stronger green fluorescence was observed at $6 \mathrm{~h}$ than that at $2 \mathrm{~h}$, indicating the internalization of nanoparticles occurred in a time-dependent manner. What's more, after incubation with BFE NPs or BFE NPs@BSA, the cytoplasm of 4T1 cells presented different intensities of coumarin-6 fluorescence. The fluorescence of cells treated with BFE NPs@BSA was much stronger than that in BFE NPs group, indicating that the surface modification of BSA could promote cellular uptake of nanoparticles.

\section{In vitro ROS generation}

It is a truism that higher than the physiological level of ROS would trigger the damage of proteins, organelles and nucleic acids, thus leading to cell apoptosis. Excessive production of ROS within cancer cells, such as superoxide anion radical $\left(\mathrm{O}_{2}{ }^{--}\right)$and hydroxyl radical $(\cdot \mathrm{OH})$, is often considered to be a 
significant condition for killing tumor cells. The designed system was supposed to produce excess ROS in multiple directions (Fig. 3D). In combination with Fe"l, BLM was transformed into an activated form and could convert oxygen into hydrogen peroxide $\left(\mathrm{H}_{2} \mathrm{O}_{2}\right)$. Highly toxic hydroxyl radicals were produced by the Fenton reactions under the high concentration of $\mathrm{H}_{2} \mathrm{O}_{2}$, leading to ROS amplification. Moreover, the transformation between $\mathrm{Fe}^{3+}$ ions and $\mathrm{Fe}^{2+}$ ions consumed glutathione heavily. This kind of GSH depletion could protect generated ROS from scavenging. Elevated temperature caused by PTT could further promote the production of ROS. Therefore, we evaluated the ROS generation ability of BFE NPs@BSA by incubation with 4T1 cancer cells. According to the fluorescence changes of a ROS-sensitive probe, 2',7'-dichlorofluorescin diacetate (DCFH-DA), the amount of generated ROS could be confirmed. As shown in Fig. 3G, weak green fluorescence (DCF) was observed in control group or cells treated with laser, indicating that ROS produced by the metabolism of cells themselves was limited. The ROS level in 4T1 cancer cells treated with BLM alone increased slightly. It was reasonable since BLM could efficiently promote the formation of hydrogen peroxide only when catalyzed by adequate iron ions. Treating 4T1 cells with BFE NPs@BSA could facilitate the generation of ROS, as an increased fluorescence signal was observed in the cytoplasm. Upon laser irradiation, bright fluorescence was observed, indicating that the significantly enhanced ROS amplification effect was triggered by NIR. Furthermore, similar results were mirrored by flow cytometry analysis which were presented in Fig. 3E and 3F. These results implied that BFE NPs@BSA combined with PTT could synergistically induce ROS amplification.

\section{In vitro relaxivity measurements and MRI performance of BFE NPs@BSA}

The incorporation of $\mathrm{Fe}^{3+}$ ions enbaled BFE NPs@BSA to be potential MRI contrast agents. To further evaluate the contrast efficacy of BFE NPs@BSA, their longitudinal relaxivity coefficient $\left(r_{1}\right)$ and transverse relaxivity coefficient $\left(r_{2}\right)$ were tested firstly by scanning BFE NPs@BSA with different concentration gradients of $\mathrm{Fe}(0,0.1,0.2,0.3,0.4$, and $0.5 \mathrm{mM})$. According to the graph data (Fig. 4A and Additional file 1: Fig. S7), BFE NPs@BSA exhibited the $\mathrm{r}_{1}$ value of $0.81 \mathrm{mM}^{-1} \mathrm{~s}^{-1}$, indicating BFE NPs@BSA were potential $\mathrm{T}_{1}$-weighted contrast agents for further application. The $\mathrm{r}_{2}$ value of BFE NPs@BSA was determined to be $2.73 \mathrm{mM}^{-1} \mathrm{~s}^{-1}$, which was too low for BFE NPs@BSA to perform as $\mathrm{T}_{2}$-weighted contrast agents. In Fig. 4B, it showed the $\mathrm{T}_{1}$-weighted images of BFE NPs@BSA at different $\mathrm{Fe}^{3+}$ concentrations, which presented positive bright contrast increasing. The MR signal was enhanced linearly with the increasing concentrations of $\mathrm{Fe}^{3+}$, indicating BFE NPs@BSA had the potential for further in vivo MR imaging.

\section{In vivo MR imaging of tumor}

It is extremely important to accurately monitor the size and location of tumor in the course of tumor therapy. Especially for photothermal or photodynamic therapy, accurate imaging of tumors can guide the course of treatment, monitor therapeutic efficacy, and reduce unexpected damage to normal tissues. MRI has become a diagnostic and research tool in treating various tumors because of its ability of accurately delineating the detailed images of the tumor tissue. As shown in Fig. 4C, 4T1 tumor-bearing BALB/C mice 
were i.v. injected with BFE NPs@BSA to assess in vivo MR imaging ability. In Fig. 4D, compared to preinjection, the tumor region of mice was significantly brighter after injection of BFE NPs@BSA, owing to the effective accumulation of BFE NPs@BSA at the tumor site. The contrast between the tumor tissue and surrounding normal tissue was more pronounced, making tumor boundary clearer. In Fig. 4E, BFE NPs@BSA was continuously enriched at the tumor site and the $T_{1}$ MR signal intensity gradually increased. At $4 \mathrm{~h}$ post-injection, the MR signal intensity of tumor site reached the maximum, which was about 1.63 times higher than that of pre-injection. After that, the MR signal intensity gradually decreased, or even fell below the value of pre-injection since $12 \mathrm{~h}$ post-injection. It might be caused by the increased tumor necrotic areas (red arrow represented the necrosis of tumor tissue), which indicated the cytotoxic effect of BFE NPs@BSA upon arrival of the tumor region. From all these results, it could reflect that effective accumulation of BFE NPs@BSA at the tumor sites could favor the precise MR imaging and cause necrosis of the tumor tissue, facilitating further therapeutic applications.

\section{Biodistribution study of BFE NPs@BSA}

To evaluate the biodistribution of BFE NPs@BSA within the body, 4T1 tumor-bearing BALB/c mice were established and tested. Fluorescent dye DiR labeled BFE NPs@BSA or free DiR were injected into tumorbearing BALB/c mice, respectively. The mice were sacrificed to obtain tumors and main organs at designed time intervals $(8,12$ and $24 \mathrm{~h}$ ). As shown in Fig. 4F, quite weak DiR fluorescence intensity was observed and remained the same level at all the time points in the tumor from mice intravenously injected with free DiR. It was similar trend as previous reports, which was ascribed to its weak tumor retention. [48] Moreover, the liver and spleen were the main accumulation organs of free DiR. By contrast, stronger fluorescence intensity was observed in the tumor from mice treated with BFE NPs@BSA. In Fig. 4G, quantification of fluorescence intensity indicated that the accumulative fluorescence intensity at the tumor site increased with prolonging time and reached the maximum fluorescence intensity at $12 \mathrm{~h}$ postinjection. The quantitative results of relative fluorescent ratio of tumor/liver displayed the same trend (Additional file 1: Fig. S8). It was worth mention that the accumulation of BFE NPs@BSA in the liver and spleen was significantly reduced, indicating that our nanomedicine was able to prolong the circulation lifetime of drugs and change metabolic pathways of drugs in the body. These results implied that BFE NPs@BSA could accumulate in the tumor region effectively.

\section{In vivo dose exploration of BLM}

Even though BLM is an effective glycopeptide anticancer drug that could affect the cutting of single- and double-stranded DNA, the risk of pulmonary fibrosis caused by BLM is still not negligible. In order to achieve the highest anti-tumor activity of BLM while minimizing its potential side effects, before applying BFE NPs@BSA into further in vivo anti-tumor experiment, the optimal dosage of BLM was firstly explored. As the experimental scheme diagram of treatment shown in Fig. 5A, 4T1 tumor-bearing BALB/c mice were i.v. injected with PBS or BFE NPs@BSA with different concentrations (BLM: 2.5, 5 and $10 \mathrm{mg} / \mathrm{kg}$ ) on day 5, 8 and 11. Both the body weight and tumor volume were measured every 2 days and tumors from mice with various treatments were separated and weighed on day 21 post-injection. As shown in Fig. 5B-E 
and Additional file 1: Fig. S9A, compared with PBS, all the groups treated with BFE NPs@BSA exhibited anti-tumor effect (inhibition rate of $47.0 \%$ for $2.5 \mathrm{mg} / \mathrm{kg}, 61.7 \%$ for $5 \mathrm{mg} / \mathrm{kg}$ and $67.7 \%$ for $10 \mathrm{mg} / \mathrm{kg}$ ) due to effective killing effect of nanoparticles on tumors.

According to the tumor growth curve, mice treated with BFE NPs@BSA (BLM: $5 \mathrm{mg} / \mathrm{kg}$ or $10 \mathrm{mg} / \mathrm{kg}$ ) exhibited similar tumor-suppressive power, as no significant difference was observed both in the tumor volume and tumor weight. But when compared to mice treated with $2.5 \mathrm{mg} / \mathrm{kg}$, better tumor inhibition with significantly smaller tumor size and low tumor weight was found in groups treated with 5 or 10 $\mathrm{mg} / \mathrm{kg}$. Then, the safety of nanomedicine was monitored by analyzing the weight of the mice and lung section. It was found that $10 \mathrm{mg} / \mathrm{kg}$ of BLM treatment gave rise to the obvious loss of body weight, while the weight of mice treated with $5 \mathrm{mg} / \mathrm{kg}$ remained unchanged. It might mean that BFE NPs@BSA (BLM: $10 \mathrm{mg} / \mathrm{kg}$ ) had overrun the safe dose. The idea was further confirmed in the results of lung section by histological analysis. As shown in Fig. 5F and S9B, the extensive inflammation was observed in the group of $10 \mathrm{mg} / \mathrm{kg}$, and this was also associated with widespread collagen (blue area by Masson's trichrome staining) accumulation and alveolar structure disorder. While the group of 2.5 and $5 \mathrm{mg} / \mathrm{kg}$ presented mild inflammation, and there were no obvious pathological changes in lung structures. BFE NPs@BSA (BLM: $10 \mathrm{mg} / \mathrm{kg}$ ) showed more severe pulmonary fibrosis of mice than other groups, which represented dose-dependent side effects. Therefore, the concentration of nanomedicine was set to BFE NPs@BSA (BLM: $5 \mathrm{mg} / \mathrm{kg}$ ) in the following experiments.

\section{In vivo anti-tumor efficiency of synergistic therapy}

To further evaluate the combined therapeutic effects of CDT and PTT, 4T1 tumor-bearing BALB/c mice were i.v. injected with PBS, free BLM ( $5 \mathrm{mg} / \mathrm{kg}$ ) or BFE NPs@BSA (BLM: $5 \mathrm{mg} / \mathrm{kg}$ ) three times on day 6, 9 and 12. Half of the mice that received BFE NPs@BSA were further locally irradiated under NIR laser every $12 \mathrm{~h}$-post injection at a power density of $2.5 \mathrm{~W} / \mathrm{cm}^{2}$ for $2 \mathrm{~min}$ (Fig. 6A). Both the body weight and tumor volume were measured every 2 days and tumors and main organs from mice with various treatments were separated and weighed on day 21. As recorded in Fig. 6B-D and 6F, BLM and BFE NPs@BSA treatment presented moderate tumor inhibitory effect comparing to PBS group. The tumor inhibitory rate of BLM and BFE NPs@BSA were 58.4\% and 69.0\%, respectively. The superior anti-tumor efficiency of BFE NPs@BSA than free BLM was attributed to the enhanced accumulation at tumor site and the ROS amplification effect of nanomedicine. Beyond expectation, the BFE NPs@BSA+L group showed the eminent inhibition compared with other groups (tumor inhibition rate was calculated to be $93.7 \%$ ) without obvious weight loss of mice. In Fig. 6E and Additional file 1: Fig. S10, complete tumor ablation was achieved in five mice of all eight mice (5/8). During the entire experimental period, the therapeutic efficacy was maintained well and no recurrence was observed in the ablated tumor. These results demonstrated the superiority of combination therapy of chemotherapy, CDT and PTT. Histological analysis of the lung sections stained with H\&E and Masson's trichrome was conducted to assess the side effects of pulmonary fibrosis. As shown in Fig. $6 \mathrm{G}$ and $6 \mathrm{H}$, severe inflammation was not observed in each group, and there were no obvious pathological changes in the lungs of all groups. In general, BFE NPs@BSA+Laser could achieve the desirable therapeutic effect with minimal toxic side effects. 


\section{Conclusions}

In summary, we constructed a metal-phenolic networks based theranostic nanoplatform through selfassembly and protein absorption. BFE NPs@BSA had great stability and photothermal conversion ability. Coordinate bonds were the driving force for the formation of BFE NPs@BSA, thus making the systems pH sensitive. In vitro and in vivo study on triple-negative breast cancer model revealed BFE NPs@BSA could achieve potent combinational therapy of chemotherapy, CDT and PTT. MRI guided individualized precise therapy might shed new light on clinical cancer treatment.

\section{Abbreviations}

TNBC: triple-negative breast cancer; MPNs: metal-phenolic networks; BLM: bleomycin; BSA: bovine serum albumin; MRI: magnetic resonance imaging; CDT: chemodynamic therapy; ROS: reactive oxygen species; TME: tumor microenvironment; $\cdot \mathrm{OH}$ : hydroxyl radicals; PTT: photothermal therapy; PTAs: photothermal transduction agents.

\section{Declarations}

\section{Ethics approval and consent to participate}

All animal experiments were conducted in accordance with the principles from the Institutional Animal Care and Use Committee (IACUC) of HUST. All animal experiments were reviewed and approved by the IACUC of HUST (IACUC Number: S2556).

\section{Consent for publication}

All authors agreed to publish this manuscript.

\section{Availability of data and materials}

All data generated or analyzed during this study are included in this article and its Additional file 1. The data used to support the findings of this study are available from the corresponding author upon reasonable request.

\section{Competing interests}

The authors declare that they have no conflict of interests.

\section{Funding}

This work was supported by National Natural Science Foundation of China $(81673374,82071890)$, Program for HUST Academic Frontier Youth Team (2018QYTD13) and Natural Science Foundation of Hubei Province (2020CFB301). 
ZP Z and LK conceived and designed the study. LK and QX wrote the original draft. QX, SL L, XX F, JY S, $Y L$ and GJ $Y$ performed the experiments. $C L Y$ and $Y Q S$ provided technical supporting during the experiment. QX, LK and ZP Z analyzed the results and revised the article. All authors read and approved the final manuscript.

\section{Acknowledgements}

We thank P. Zhang and A. Du from The Core Facility and Technical Support, Wuhan Institute of Virology, for performing the TEM (JEM-1230, Japan).

\section{Authors' information}

${ }^{1}$ Tongji School of Pharmacy, Huazhong University of Science and Technology, Wuhan 430030, China.

${ }^{2}$ Department of radiology, Tongji Hospital of Tongji Medical College of Huazhong University of Science and Technology, Wuhan 430030, China. ${ }^{3}$ Liyuan Hospital of Tongji Medical College of Huazhong University of Science and Technology, Wuhan 430030, China. ${ }^{4}$ National Engineering Research Center for Nanomedicine, Huazhong University of Science and Technology, Wuhan, 430030, China. ${ }^{5}$ Hubei Engineering Research Center for Novel Drug Delivery System, Huazhong University of Science and Technology, Wuhan, 430030, China.

\section{References}

1. Siegel RL, Miller KD, Fuchs HE, Jemal A: Cancer Statistics, 2021. CA Cancer J Clin 2021, 71:7-33.

2. DeSantis CE, Ma J, Gaudet MM, Newman LA, Miller KD, Goding Sauer A, Jemal A, Siegel RL: Breast cancer statistics, 2019. CA Cancer J Clin 2019, 69:438-451.

3. Waks AG, Winer EP: Breast Cancer Treatment: A Review. JAMA 2019, 321:288-300.

4. Carey L, Winer E, Viale G, Cameron D, Gianni L: Triple-negative breast cancer: disease entity or title of convenience? Nat Rev Clin Oncol 2010, 7:683-692.

5. Bianchini G, Balko JM, Mayer IA, Sanders ME, Gianni L: Triple-negative breast cancer: challenges and opportunities of a heterogeneous disease. Nat Rev Clin Oncol 2016, 13:674-690.

6. Shu G, Chen M, Song J, Xu X, Lu C, Du Y, Xu M, Zhao Z, Zhu M, Fan K, et al: Sialic acid-engineered mesoporous polydopamine nanoparticles loaded with $\mathrm{SPIO}$ and $\mathrm{Fe}(3+)$ as a novel theranostic agent for T1/T2 dual-mode MRI-guided combined chemo-photothermal treatment of hepatic cancer. Bioact Mater 2021, 6:1423-1435.

7. Carey LA, Dees EC, Sawyer L, Gatti L, Moore DT, Collichio F, Ollila DW, Sartor Cl, Graham ML, Perou CM: The triple negative paradox: primary tumor chemosensitivity of breast cancer subtypes. Clin Cancer Res 2007, 13:2329-2334. 
8. Denkert C, Liedtke C, Tutt A, von Minckwitz G: Molecular alterations in triple-negative breast cancerthe road to new treatment strategies. The Lancet 2017, 389:2430-2442.

9. Kalimutho M, Parsons K, Mittal D, López JA, Srihari S, Khanna KK: Targeted Therapies for TripleNegative Breast Cancer: Combating a Stubborn Disease. Trends in Pharmacological Sciences 2015, 36:822-846.

10. Hu H, Feng W, Qian X, Yu L, Chen Y, Li Y: Emerging Nanomedicine-Enabled/Enhanced Nanodynamic Therapies beyond Traditional Photodynamics. Adv Mater 2021:e2005062.

11. Li SL, Jiang P, Jiang FL, Liu Y: Recent Advances in Nanomaterial-Based Nanoplatforms for Chemodynamic Cancer Therapy. Advanced Functional Materials 2021, 31:2100243.

12. Zhong X, Wang $X$, Li J, Hu J, Cheng L, Yang X: ROS-based dynamic therapy synergy with modulating tumor cell-microenvironment mediated by inorganic nanomedicine. Coordination Chemistry Reviews 2021, 437:213828.

13. Tang W, Gao H, Ni D, Wang Q, Gu B, He X, Peng W: Bovine serum albumin-templated nanoplatform for magnetic resonance imaging-guided chemodynamic therapy. J Nanobiotechnology 2019, 17:68.

14. Zhao B, Zhao P, Jin Z, Fan M, Meng J, He Q: Programmed ROS/CO-releasing nanomedicine for synergetic chemodynamic-gas therapy of cancer. J Nanobiotechnology 2019, 17:75.

15. Tang Z, Liu Y, He M, Bu W: Chemodynamic Therapy: Tumour Microenvironment-Mediated Fenton and Fenton-like Reactions. Angew Chem Int Ed Eng/2019, 58:946-956.

16. Qian X, Zhang J, Gu Z, Chen Y: Nanocatalysts-augmented Fenton chemical reaction for nanocatalytic tumor therapy. Biomaterials 2019, 211:1-13.

17. Wang W, Jin Y, Xu Z, Liu X, Bajwa SZ, Khan WS, Yu H: Stimuli-activatable nanomedicines for chemodynamic therapy of cancer. Wiley Interdiscip Rev Nanomed Nanobiotechno/ 2020, 12:e1614.

18. Wang X, Zhong X, Liu Z, Cheng L: Recent progress of chemodynamic therapy-induced combination cancer therapy. Nano Today 2020, 35:100946.

19. Yao J, Zheng F, Yao C, Xu X, Akakuru OU, Chen T, Yang F, Wu A: Rational design of nanomedicine for photothermal-chemodynamic bimodal cancer therapy. Wiley Interdiscip Rev Nanomed Nanobiotechno/ 2020:e1682.

20. Zou L, Wang H, He B, Zeng L, Tan T, Cao H, He X, Zhang Z, Guo S, Li Y: Current Approaches of Photothermal Therapy in Treating Cancer Metastasis with Nanotherapeutics. Theranostics 2016, 6:762-772.

21. Liu Y, Bhattarai P, Dai Z, Chen X: Photothermal therapy and photoacoustic imaging via nanotheranostics in fighting cancer. Chem Soc Rev 2019, 48:2053-2108.

22. Liu J, Shi J, Nie W, Wang S, Liu G, Cai K: Recent Progress in the Development of Multifunctional Nanoplatform for Precise Tumor Phototherapy. Adv Healthc Mater 2021, 10:e2001207.

23. Schoenfeld JD, Sibenaller ZA, Mapuskar KA, Wagner BA, Cramer-Morales KL, Furqan M, Sandhu S, Carlisle TL, Smith MC, Abu Hejleh T, et al: 02(-) and H2O2-Mediated Disruption of Fe Metabolism 
Causes the Differential Susceptibility of NSCLC and GBM Cancer Cells to Pharmacological Ascorbate. Cancer Cel/ 2017, 31:487-500 e488.

24. Dixon SJ, Lemberg KM, Lamprecht MR, Skouta R, Zaitsev EM, Gleason CE, Patel DN, Bauer AJ, Cantley AM, Yang WS, et al: Ferroptosis: an iron-dependent form of nonapoptotic cell death. Cell 2012, 149:1060-1072.

25. Dixon SJ, Stockwell BR: The role of iron and reactive oxygen species in cell death. Nat Chem Biol 2014, 10:9-17.

26. Ejima H, Richardson JJ, Liang K, Best JP, van Koeverden MP, Such GK, Cui J, Caruso F: One-Step Assembly of Coordination Complexes for Versatile Film and Particle Engineering. Science 2013, 341:154-157.

27. Zhou J, Lin Z, Ju Y, Rahim MA, Richardson JJ, Caruso F: Polyphenol-Mediated Assembly for Particle Engineering. Acc Chem Res 2020, 53:1269-1278.

28. Liu F, He X, Chen H, Zhang J, Zhang H, Wang Z: Gram-scale synthesis of coordination polymer nanodots with renal clearance properties for cancer theranostic applications. Nat Commun 2015, 6:8003.

29. Ls A, Ty A, Cy A, Zhen LB, Li KA, Zzac D: Metal ions-mediated self-assembly of nanomedicine for combinational therapy against triple-negative breast cancer. Chemical Engineering Journal 2021, 425:131420.

30. Rahim MA, Kristufek SL, Pan S, Richardson JJ, Caruso F: Phenolic Building Blocks for the Assembly of Functional Materials. Angew Chem Int Ed Engl 2019, 58:1904-1927.

31. Dai Q, Geng H, Yu Q, Hao J, Cui J: Polyphenol-Based Particles for Theranostics. Theranostics 2019, 9:3170-3190.

32. Xie W, Guo Z, Zhao L, Wei Y: Metal-phenolic networks: facile assembled complexes for cancer theranostics. Theranostics 2021, 11:6407-6426.

33. Zhang P, Hou Y, Zeng J, Li Y, Wang Z, Zhu R, Ma T, Gao M: Coordinatively Unsaturated Fe(3+) Based Activatable Probes for Enhanced MRI and Therapy of Tumors. Angew Chem Int Ed Eng/2019, 58:11088-11096.

34. Liu T, Zhang M, Liu W, Zeng X, Song X, Yang X, Zhang X, Feng J: Metal lon/Tannic Acid Assembly as a Versatile Photothermal Platform in Engineering Multimodal Nanotheranostics for Advanced Applications. ACS Nano 2018, 12:3917-3927.

35. Zhang C, Li J, Yang C, Gong S, Jiang H, Sun M, Qian C: A pH-sensitive coordination polymer networkbased nanoplatform for magnetic resonance imaging-guided cancer chemo-photothermal synergistic therapy. Nanomedicine 2020, 23:102071.

36. Stockwell BR, Friedmann Angeli JP, Bayir H, Bush Al, Conrad M, Dixon SJ, Fulda S, Gascon S, Hatzios SK, Kagan VE, et al: Ferroptosis: A Regulated Cell Death Nexus Linking Metabolism, Redox Biology, and Disease. Cell 2017, 171:273-285.

37. Guo Y, Sun Q, Wu FG, Dai Y, Chen X: Polyphenol-Containing Nanoparticles: Synthesis, Properties, and Therapeutic Delivery. Adv Mater 2021, 33:e2007356. 
38. Chen J, Stubbe J: Bleomycins: towards better therapeutics. Nat Rev Cancer 2005, 5:102-112.

39. Basu U, Roy M, Chakravarty AR: Recent advances in the chemistry of iron-based chemotherapeutic agents. Coordination Chemistry Reviews 2020, 417:213339.

40. Bugaut $H$, Bruchard $M$, Berger $H$, Derangere V, Odoul L, Euvrard $R$, Ladoire $S$, Chalmin F, Vegran $F$, Rebe $\mathrm{C}$, et al: Bleomycin exerts ambivalent antitumor immune effect by triggering both immunogenic cell death and proliferation of regulatory T cells. PLOS One 2013, 8:e65181.

41. Burgy O, Wettstein G, Bellaye PS, Decologne N, Racoeur C, Goirand F, Beltramo G, Hernandez J-F, Kenani A, Camus $\mathrm{P}$, et al: Deglycosylated bleomycin has the antitumor activity of bleomycin without pulmonary toxicity. Science Translational Medicine 2016, 8.

42. Yu Z, Yan B, Gao L, Dong C, Zhong J, M DO, Nguyen B, Seong Lee S, Hu X, Liang F: Targeted Delivery of Bleomycin: A Comprehensive Anticancer Review. Curr Cancer Drug Targets 2016, 16:509-521.

43. Feng Q, Zhang W, Li Y, Yang X, Hao Y, Zhang H, Li W, Hou L, Zhang Z: An intelligent NIR-responsive chelate copper-based anticancer nanoplatform for synergistic tumor targeted chemo-phototherapy. Nanoscale 2017, 9:15685-15695.

44. Koshkaryev A, Piroyan A, Torchilin VP: Bleomycin in octaarginine-modified fusogenic liposomes results in improved tumor growth inhibition. Cancer Lett 2013, 334:293-301.

45. Georgelin T, Bombard S, Siaugue JM, Cabuil V: Nanoparticle-mediated delivery of bleomycin. Angew Chem Int Ed Engl 2010, 49:8897-8901.

46. Han Y, Lin Z, Zhou J, Yun G, Guo R, Richardson JJ, Caruso F: Polyphenol-Mediated Assembly of Proteins for Engineering Functional Materials. Angew Chem Int Ed Eng/2020, 59:15618-15625.

47. Taha MS, Cresswell GM, Park J, Lee W, Ratliff TL, Yeo Y: Sustained Delivery of Carfilzomib by Tannic Acid-Based Nanocapsules Helps Develop Antitumor Immunity. Nano Lett 2019, 19:8333-8341.

48. Tu K, Deng H, Kong L, Wang Y, Yang T, Hu Q, Hu M, Yang C, Zhang Z: Reshaping Tumor Immune Microenvironment through Acidity-Responsive Nanoparticles Featured with CRISPR/Cas9-Mediated Programmed Death-Ligand 1 Attenuation and Chemotherapeutics-Induced Immunogenic Cell Death. ACS Appl Mater Interfaces 2020, 12:16018-16030.

\section{Schema}

Schema 1 available in supplementary section

\section{Figures}

Figure 1

(A) Schematic description of BFE NPs preparation. The particle size and zeta potential of BFE NPs at different molar ratios of EGCG (B) and $\mathrm{Fe}^{3+}(\mathrm{C})$. The particle size, PDI (D) and photograph (E) of BFE NPs 
in various media. (F) The long-term stability of BFE NPs. (G) Schematic description of BFE NPs@BSA preparation. $(\mathrm{H})$ The particle size and PDI of nanoparticles at different BSA concentrations. (I) The longterm stability of BFE NPs@BSA. (J) The summary of the four types of nanoparticles. The TEM images of BFE NPs (K) and BFE NPs@BSA (L). Scale bars were 200 nm.

\section{Figure 2}

The UV-vis spectra (A), fluorescent spectra (B) and FT-IR spectra (C) analysis. (D) The photographs of BFE NPs and BFE NPs@BSA dissociation by NaCl, Urea, EDTA and SDS. (E) The fluorescence recovery analysis of BFE NPs@BSA after treatment with EDTA at different time points. (F) The changes of particle size of BFE NPs@BSA in PBS at different pH value. The fluorescence recovery analysis of BFE NPs@BSA in PBS at pH $6.5(\mathrm{G})$ and pH $5.5(\mathrm{H})$. (I) The drug release behavior of BLM in PBS at different pH value. (J) The temperature elevation of BFE NPs@BSA under $808 \mathrm{~nm}$ laser irradiation with different power density for 10 min at $\mathrm{Fe}^{3+}$ concentration of $0.8 \mathrm{mM}$. (K) The temperature elevation of BFE NPs@BSA under laser irradiation $\left(808 \mathrm{~nm}, 2.5 \mathrm{~W} / \mathrm{cm}^{2}\right)$ for $10 \mathrm{~min}$ at different $\mathrm{Fe}^{3+}$ concentrations. (L) The temperature changes of BFE NPs@BSA within 5 laser-on and off cycles.

\section{Figure 3}

(A) The viability of $4 T 1$ cells after different treatments $(n=6)$. (B) 4T1 cells stained by Calcein-AM/PI kit after different treatments. Scale bars were $20 \mu \mathrm{m}$. (C) The cellular uptake of coumarin-6 labeled BFE NPs and BFE NPs@BSA. Scale bars were $20 \mu \mathrm{m}$. (D) Schematic illustration of intracellular mechanisms. (E) The flow cytometry analysis of ROS generation after different treatments and (F) corresponding MFI values $(n=3)$. $(G)$ The fluorescence images of ROS probes after different treatments. Scale bars were 50 $\mu \mathrm{m}$. Statistical $p$ values verse the control group: ${ }^{*} p<0.05,{ }^{\star \star} p<0.01,{ }^{\star \star \star} p<0.001$.

\section{Figure 4}

(A) The linear fitting of $1 / T_{1}$ of BFE NPs@BSA at different Fe ${ }^{3+}$ concentrations. (B) The $T_{1}$-weighted MR images of BFE NPs@BSA nanoparticle. (C) Schematic description of MR scanning plan. (D) The in vivo $T_{1} M R$ images of mice at different time points. (E) The quantitative analysis of relative $T_{1}$ signal of tumor region $(n=6)$. (F) The in vivo biodistribution of the DiR-loaded BFE NPs@BSA $(n=3) .(G)$ The quantitative analysis of fluorescence intensity in tumor. Statistical $p$ values: ${ }^{\star} p<0.05,{ }^{\star \star} p<0.01,{ }^{\star \star *} p$ $<0.001$. 


\section{Figure 5}

(A) Schematic diagram of 4T1 tumor treatment. The 4T1 tumor growth curve (B), body weight of mice (C) and tumor weight $(D)$ after different treatments $(n=5)$. (E) The photograph of tumors after different treatments. (F) H\&E and masson's trichrome staining of the lung sections from the mice after different treatments. Statistical $p$ values: ${ }^{\star} p<0.05, * \star p<0.01, * \star * p<0.001$.

\section{Figure 6}

(A) Schematic diagram of $4 \mathrm{~T} 1$ tumor treatment. The $4 \mathrm{~T} 1$ tumor growth curve (B) tumor weight (C) and tumor inhibition rate $(D)$ after different treatments $(n=8)$. (E) The photograph of tumors after different treatments. (F) The body weight of mice after different treatments. $H \& E(G)$ and masson's $(H)$ trichrome staining of the lung sections from the mice after different treatments. Statistical $p$ values: ${ }^{\star} p<0.05, * \star p$ $<0.01, \star \star \star p<0.001$.

\section{Supplementary Files}

This is a list of supplementary files associated with this preprint. Click to download.

- 2021.12.15Additionalfile.docx

- 12.15Graphicalabstractimage.tif

- schema1.png 